\title{
FATORES DE RISCO PARA DOENÇA ARTERIAL CORONARIANA EM IDOSOS: ANÁLISE POR ENFERMEIROS UTILIZANDO FERRAMENTA COMPUTACIONAL
}

\author{
Risk factors for coronary artery disease in the elderly: analysis by nurses \\ using computational tool
}

\author{
Factores de riesgo para enfermedad arterial coronaria en ancianos: análisis por \\ enfermeras utilizando herramienta computacional
}

\section{RESUMO}

Este trabalho objetivou analisar a ocorrência dos fatores de risco para doença arterial coronariana em população idosa participante de uma ação comunitária utilizando ferramenta computacional por enfermeiros. Para o desenvolvimento do trabalho utilizou-se uma base de dados coletada em um evento comunitário. As informaç̧ões se referem a fatores de risco, dados antropométricos, aferição de valores de glicemia, colesterol e pressão arterial, ocorrência de doença cardíaca e outras. A estrutura multidimensional foi elaborada e gerenciada pela ferramenta Analysis Services. A população idosa correspondeu a $40,4 \%$ do total, um terço dessa população estava com valores alterados de pressão arterial sistêmica, 53,8\% apresentavam índice de massa corporal acima de $25 \mathrm{Kg} / \mathrm{m}^{2}, 40,3 \%$ referiram hipertensão e $20,3 \%$, diabetes mellitus. Conclui-se que o controle de fatores de risco para DAC em clientes idosos é essencial e que a tecnologia da informação pode apoiar na tomada de decisões estratégicas de promoção de saúde.

Palavras-chave: Saúde do Idoso. Doença da Artéria Coronariana. Técnicas de Apoio para a Decisão. Fatores de Risco. Enfermagem.

\begin{abstract}
This study aimed to investigate the occurrence of risk factors for coronary artery disease among the elderly participants in a community campaign using a computational tool by nurses. In order to carry out the investigation, a database built during a particular event in the community has been used. The information concerns risk factors; anthropometric data; readings of glucose, cholesterol and blood pressure levels; occurrence of cardiac disease and others. The multidimensional structure was generated and handled by the Analysis Services tool. The elderly population represented $40.4 \%$ of the total, and one-third of that population showed altered levels of systemic blood pressure, 53.8\% presented body mass index above $25 \mathrm{Kg} / \mathrm{m}^{2}, 40.3 \%$ reported hypertension and $20.3 \%$ diabetes mellitus. It can be inferred that it is essential to control risk factors for CAD among the elderly, and that information technology can be useful to make strategic decisions in health care promotion.
\end{abstract}

Keywords: Health of the Elderly. Coronary Artery Disease. Decision Support Techniques. Risk Factors. Nursing.

\section{Resumen}

Este trabajo tuvo como objetivo analizar la ocurrencia de los factores de riesgo para enfermedad arterial coronaria en la población anciana participante de una acción comunitaria con una herramienta computacional para las enfermeras. Para el desarrollo del trabajo se utilizó una base de datos colectada en un evento comunitario. Las informaciones se refieren a factores de riesgo, datos antropométricos, contraste de valores de glucemia, colesterol y presión arterial, ocurrencia de enfermedad cardiaca yotras. La estructura multidimensional fue elaborada y administrada por la herramienta Analysis Services. La población anciana correspondió a 40,4\% del total, un tercio de esta población estaba con valores alterados de presión arterial sistémica, 53,8\% presentaban índice de masa corporal arriba de $25 \mathrm{Kg} / \mathrm{m}^{2}, 40,4 \%$ se referían a hipertensión y $20,3 \%$ diabetes mellitus. Se concluye que el control de factores de riesgo para DAC en clientes ancianos es esencial y que la tecnología de la información puede apoyar en la tomada de decisiones estratégicas de promoción de salud.

Palabras clave: Salud del Anciano. Enfermedad de la Arteria Coronaria. Técnicas de Apoyo para la Decisión. Factores de Riesgo. Enfermería.

'Doutora Professora e Coordenadora do Curso de Graduação em Enfermagem, Universidade de Ribeirão Preto, Ribeirão Preto -SP. Brasil. E-mail: sssilva@unaerp.br, ${ }^{2}$ Doutor Professor e Coordenador dos Cursos de Engenharia de Computação e Sistemas de Informação, Centro de Ciências Exatas Naturais e Tecnológicas, Universidade de Ribeirão Preto, Ribeirão Preto-SP. Brasil. E-mail: ecarita@unaerp.br, ${ }^{3}$ Especialista Professora do Curso de Especialização em Banco de Dados, Centro de Ciências Exatas Naturais e Tecnológicas, Universidade de Ribeirão Preto, Ribeirão Preto-SP. Brasil. E-mail: elianardiniz@hotmail.com 


\section{INTRODUÇÃO}

A primeira edição da ação comunitária Unaerp Solidária realizou-se no ano de 2004 em comemoração aos 80 anos da Universidade de Ribeirão Preto e ao aniversário de 148 anos da cidade de Ribeirão Preto-SP, oferecendo 40 tipos de atendimentos nas mais diversas áreas, além de apresentações musicais e de dança, com o objetivo de aproximar a universidade da população. Desde então, o evento vem ocorrendo anualmente, e, no ano de 2009, dentre as ações citadas, os profissionais dos cursos de Enfermagem, Engenharia de Computação, Engenharia de Produção e Sistemas de Informação desenvolveram um questionário eletrônico para coletar dados referentes à saúde da população, com a finalidade de levantar os fatores de risco para a doença arterial coronariana, especialmente em idosos.

A população brasileira está envelhecendo de forma acentuada, de acordo com dados do Instituto Brasileiro de Geografia e Estatística (IBGE) ${ }^{1}$ sinalizando que a atual expectativa de vida é de 72,7 anos. A Organização Mundial de Saúde (OMS) classifica o indivíduo idoso como aquele de 65 anos ou mais de idade, em países desenvolvidos, e 60 anos ou mais para pessoas de países subdesenvolvidos. Entende-se que envelhecer é processo natural no ciclo vital; porém, compartilhase com pesquisadores da área que existe um contraponto entre o aumento da população idosa e o suporte necessário para atendimento às pessoas pertencentes a essa faixa etária. ${ }^{2}$

Estudiosos da temática salientam que, embora se evidenciem inúmeros avanços nas áreas de saúde e tecnologia que priorizam o prolongamento da vida do ser humano, o que realmente se constata no meio social é o aumento exacerbado do sofrimento, falta de informação e restrição no acesso aos serviços de apoio social e de saúde, configurando um contrassenso quando se identifica que a longevidade não ocorre aliada a qualidade de vida. ${ }^{3}$

0 envelhecimento deve ser compreendido como um processo não patológico, com diminuição progressiva da reserva funcional. Todavia, em condições de sobrecarga, como na vigência de doenças cardiovasculares e estresse emocional, podem-se instalar quadros patológicos que exigem assistência, caracterizando a senilidade. ${ }^{4}$ Essa condição de debilidade física ou intelectual pode ser evitada quanto menor for a exposição aos fatores de risco para as doenças cardiovasculares, por exemplo.

Nessa direção, existem alguns respaldos como as políticas públicas voltadas à pessoa idosa que contempla leis, Estatuto do Idoso, entre outras providências que conferem alguma proteção àqueles que chegam à idade avançada, nos âmbitos social, político e de saúde; porém, muito há para se fazer quanto à inclusão adequada da pessoa idosa na sociedade, e, entre os aspectos a serem considerados, a Enfermagem tem se deparado com a necessidade de levar/oferecer mais informações de forma rápida, prática e clara a esses consumidores de saúde, apresentando profissionais promotores de mudanças na equipe e na comunidade. ${ }^{5}$

Ressalta-se, diante do exposto, que o envelhecimento da população brasileira necessita de um diagnóstico de saúde nas esferas nacional, regional e local, que possibilite conduzir propostas realistas; nesse sentido, justifica-se a coleta de dados proposta nesse trabalho.

Atualmente, decisões estratégicas são de grande importância para todas as áreas do saber, seja para conquistar clientes, recursos financeiros ou melhorar a qualidade de vida das pessoas. Na saúde, a utilização de um sistema de apoio à tomada de decisões pode ser de grande auxílio, permitindo a análise de dados através da correlação de informações, relacionando problemas de saúde com seus fatores determinantes e identificando os riscos para acometimento de doenças. Os indicadores deverão servir de elementos para a execução de ações de prevenção para melhorar a qualidade de vida dos clientes/usuários. ${ }^{6}$

Este estudo tem o objetivo de analisar a ocorrência dos fatores de risco para doença arterial coronariana em população idosa participante de uma ação comunitária, utilizando ferramenta computacional por enfermeiros.

\section{REVISÃO DE LITERATURA}

Historicamente, a visão do idoso em diferentes sociedades está permeada por vários aspectos, e reconhecese a dificuldade de determinar quem é o idoso, respaldandose, muitas vezes, no critério etário para essa definição. 0 envelhecimento é compreendido a partir de diversas concepções culturais referindo-se a processos biológicos, aparência física, eventos de desengajamento da vida social e 0 aparecimento de novos papéis sociais.?

A Organização Pan-Americana de Saúde (OPAS) ${ }^{8}$ define o envelhecimento do ponto de vista fisiológico como um processo cumulativo, irreversível e universal, não patológico, em que ocorre a deterioração do organismo maduro, podendo levar a algumas incapacitações.

0 índice de envelhecimento, uma medida que considera apenas os dois grupos etários extremos, aqueles mais afetados no processo de envelhecimento, mostra que em 2025, provavelmente, estará três vezes maior do que aquele observado em $2000 .{ }^{9} \mathrm{Na}$ população brasileira teremos mais de 50 adultos com 65 anos ou mais por cada conjunto de 100 jovens menores de 15 anos. Em 2045, o número de pessoas idosas ultrapassaria o de crianças. ${ }^{9}$

Esse crescimento é decorrente da combinação de fatores que envolvem esse processo, podendo ter como causas os avanços tecnológicos e das ciências médicas e as reduções das taxas de natalidade e mortalidade, resultando em aumento na expectativa de vida para 81,3 anos em 2050, de acordo com o IBGE. 
Além dessa constatação, verifica-se que há um aumento das doenças crônicas não transmissíveis, característica do perfil populacional idoso, que pode interferir no nível de dependência e independência dessas pessoas. Estudos revelam que a cardiopatia isquêmica representa a principal causa de óbito no idoso, com prevalência de $70 \%$ de doença arterial coronariana (DAC) nesta faixa etária e $20 \%$ a $30 \%$ dos idosos com manifestações clínicas de doença isquêmica. ${ }^{10}$

Ao se tornar epidemia nos Estados Unidos da América, a DAC mereceu investigação visando detecção de suas causas, controle e educação preventiva. 0 termo fator de risco surgiu, em 1963, em um artigo médico, denominado "Fatores de risco em doença cardíaca coronariana". 0 desenvolvimento do conceito de fatores de risco e suas relações com a incidência de coronariopatias decorreram de estudos epidemiológicos prospectivos realizados nos Estados Unidos ${ }^{11}$, quando se estabeleceu a relação entre estilo de vida e DAC.

Desde o primeiro relato no estudo de Framingham, destacou-se que os fatores de risco pareciam estar ligados ao surgimento da doença aterosclerótica, e, assim, traduziram-se em condições que podem predizer a possibilidade de um indivíduo desenvolver determinada doença. ${ }^{11}$

Nesse sentido, os fatores de risco cardiovasculares relacionados à aterosclerose podem ser divididos de acordo com suas possibilidades de modificação, seja através de mudanças de comportamento ou tipos de tratamento indicados. Assim, têm-se os grupos de fatores de risco modificáveis e os não modificáveis. ${ }^{12}$

Os fatores de risco modificáveis são aqueles controláveis, podendo ser controlados e até excluídos; incluindo tabagismo, hipertensão arterial sistêmica, a intolerância à glicose e diabetes mellitus não insulinodependente, sedentarismo, obesidade, uso de anticoncepcionais hormonais, estresse e dislipidemia. ${ }^{12}$ Já os fatores não modificáveis são aqueles nos quais não se pode exercer influência no sentido de mudá-los e, desta forma, não podem ser modificados ou excluídos compreendendo a etnia, sexo e idade; história familiar e ocorrência da menopausa. ${ }^{12}$

Assim, éfundamental evitar e/ou minimizar a exposição aos fatores de risco para a ocorrência da DAC nessa população, identificando-os e discutindo sobre sua importância com a comunidade idosa por meio de programas educativos, uma vez que 0 aspecto educacional tem se apresentado fundamental para a diminuição da ocorrência dos fatores de risco para a DAC e suas implicações na vida dos clientes/usuários.

\section{MÉTODO}

Para a análise dos resultados foi estruturado um Data Mart acessado por ferramentas On-Line Analytical Processing $(\mathrm{OLAP})^{\mathrm{b}}$ com o objetivo de extrair informações referentes à análise de ocorrência de fatores de risco para a doença arterial coronariana em população idosa.
0 desenvolvimento do trabalho originou-se de uma base de dados coletada no evento Unaerp Solidária 2009, composto por 1.770 pessoas na faixa etária de 12 a 90 anos, excluindo os sujeitos menores de 12 anos. Os dados foram coletados por profissionais treinados da área de saúde e exatas utilizando um sistema de informação em saúde, em espaço privativo. As informações levantadas referem-se a fatores de risco modificáveis e não modificáveis, dados antropométricos, aferição de valores de glicemia, colesterol e pressão arterial, ocorrência de doença cardíaca e outras. A população idosa constituiu-se de 715 indivíduos que assinaram, individualmente, um termo de consentimento livre e esclarecido, após ter ciência do objetivo da pesquisa, atendendo a Resolução 196/96 do Conselho Nacional de Saúde, com autorização pelo Comitê de Ética em Pesquisa com Seres Humanos da Universidade de Ribeirão Preto, sob protocolo n ${ }^{0}$ 062/08.

As informações coletadas no referido evento foram armazenadas no Sistema Gerenciador de Banco de Dados (SGBD) Microsoft SQL Server Express.

A estrutura multidimensional, que contém detalhes e dados de agregação em um único modelo lógico e unificado, com suporte para cálculos internos, foi projetada, criada e gerenciada por meio da ferramenta Analysis Services (MSSQL Server). 0 esquema de estrutura usado neste projeto foi o modelo estrela que define os elementos de composição do cubo.

0 processamento do cubo baseado em estrutura multidimensional armazena as informações calculando previamente todas as combinações de todos os níveis de dimensão e agregação, gerando um esquema otimizado para que as consultas realizadas sejam respondidas rapidamente. Foi escolhida a forma de armazenamento MOLAP (OLAP Multidimensional - onde os dados detalhados e agregações são armazenados em formato de cubo do Analysis Server). Considerando que os dados armazenados estão em uma única base de dados relacional normalizada, não foram necessárias a transformação e a limpeza dos dados, pois não havia dados nulos e/ou inválidos.

Para disponibilizar a visualização dos dados do cubo foi utilizado o componente de tabela dinâmica e gráfico (do Analysis Server), estabelecendo uma conexão com a fonte de dados multidimensional.

A tabela dinâmica, sendo uma ferramenta gráfica, permite que os usuários explorem facilmente as dimensões e as medidas do cubo, elaborando uma hipótese e obtendo os resultados através da correlação de variáveis. Várias operações são possíveis, como aumentar ou diminuir o nível de detalhe, agrupar as informações, além de alterar linhas e colunas modificando as visões. 


\section{RESULTADOS E DISCUSSÃO}

A amostra constituiu-se de 1.770 indivíduos, sendo $1.108(62,6 \%)$ do sexo feminino e $662(37,4 \%)$, do sexo masculino. A população idosa compunha-se de 715 indivíduos, o que corresponde a $40,4 \%$ da população total, sendo 444 $(62,1 \%)$ do sexo feminino e $271(37,9 \%)$ do sexo masculino.

Considerando a população idosa, 286 indivíduos tiveram a pressão arterial aferida, e 99 indivíduos (34,6\%) apresentaram valores anormais (igual ou maior que $140 \times 90 \mathrm{mmHg}$ ), de acordo com o preconizado na V Diretrizes Brasileiras de Hipertensão Arterial, ${ }^{13}$ sendo 67 indivíduos do sexo feminino e 32 indivíduos do sexo masculino, com apenas $13,1 \%$ dos sujeitos relatando ter doença cardíaca. Observase, pelos achados, que um terço da população idosa atendida estava com valores alterados de pressão arterial sistêmica.

Em relação ao Índice de Massa Corporal (IMC), segundo a classificação da International Lipid Information Bureau Latino America $^{14}, 53,8 \%$ dos clientes apresentavam índice de massa corporal acima de $25 \mathrm{Kg} / \mathrm{m}^{2}$, com 33,7\% idosos apresentando sobrepeso, sendo 19,2\% mulheres e 13,9\% homens. Destes, $15,4 \%$ das mulheres apresentaram valores de colesterol total acima de $200 \mathrm{mg} / \mathrm{dL}$ contra 5,7\% dos homens. As mulheres ainda apresentaram obesidade grau $\mathrm{I}$ e II, com percentuais de $23,3 \%$ e $18,2 \%$, respectivamente.

Considerando os valores glicêmicos adotados pela American Diabetes Association ${ }^{15}$ e a presença de IMC alterado, observou-se que $14,2 \%$ dos idosos tinham alteração desses índices, sendo que na faixa de 100 a $125 \mathrm{mg} / \mathrm{dL}$ havia $34,3 \%$ mulheres e 2,9\% homens com sobrepeso; $25,6 \%$ homens e mulheres com obesidade grau I, e 21,6\% mulheres e 11,8\% homens com obesidade grau II. Com valores glicêmicos acima de $125 \mathrm{mg} / \mathrm{dL}$, apresentaram-se $66,7 \%$ mulheres e $8,3 \%$ homens.

Na busca de identificar idosos com valores de pressão arterial, glicemia e colesterol total alterados, considerando os mesmos referenciais que nortearam as análises nesse estudo, constatou-se que apenas as mulheres (3,9\%) apresentaram a combinação desses valores alterados na amostra, sendo que $2,6 \%$ delas tinham glicemia acima de $125 \mathrm{mg} / \mathrm{dL}$ (Figura 1); porém, ao levantar a presença de fatores não modificáveis indicados pelos idosos, verificou-se que uma pessoa do sexo masculino tinha, além dos valores supracitados alterados, também o hábito de fumar.

Figura 1: Consulta apresentando os valores de Colesterol, Glicemia e Pressão Arterial Sistêmica das pessoas com faixa etária acima de 60 anos.

\begin{tabular}{|c|c|c|c|c|c|c|c|}
\hline \multirow[b]{3}{*}{ Faixa Etária * } & \multirow[b]{3}{*}{ Colesterol } & \multirow[b]{3}{*}{ Glicemia } & \multirow[b]{3}{*}{ Sexo } & \multicolumn{4}{|c|}{ Pressão Arterial * } \\
\hline & & & & \multicolumn{2}{|c|}{$<140 \times 90 \mathrm{mmHg}$ - (valores normais) } & \multicolumn{2}{|c|}{$=140 \times 90 \mathrm{mmHg}$ (valores anormais) } \\
\hline & & & & Qtde & Percentual & Qtde & tual \\
\hline \multirow[t]{13}{*}{ 曰60 Anos ou + } & \multirow[t]{6}{*}{$\boxminus$ Acima de $200 \mathrm{mg} / \mathrm{dL}$} & \multirow[t]{2}{*}{$\boxminus 70$ a $99 \mathrm{mg} / \mathrm{dL}$} & $\mathbf{F}$ & 7 & $4,46 \%$ & 8 & $10,39 \%$ \\
\hline & & & M & 3 & $1,91 \%$ & 2 & $2,60 \%$ \\
\hline & & \multirow[t]{2}{*}{$\boxminus 100$ a $125 \mathrm{mg} / \mathrm{dL}$} & $\mathbf{F}$ & 11 & $7,01 \%$ & 1 & $1,30 \%$ \\
\hline & & & M & 4 & $2,55 \%$ & & \\
\hline & & 曰Acima $125 \mathrm{mg} / \mathrm{dL}$ & $\mathbf{F}$ & 4 & $2,55 \%$ & 2 & $2,60 \%$ \\
\hline & & Total & & 29 & $18,47 \%$ & 13 & $16,88 \%$ \\
\hline & \multirow[t]{7}{*}{ 日 Ate $200 \mathrm{mg} / \mathrm{dL}$} & \multirow[t]{2}{*}{$\boxminus 70$ a $99 \mathrm{mg} / \mathrm{dL}$} & $\mathbf{F}$ & 46 & $29,30 \%$ & 16 & $20,78 \%$ \\
\hline & & & M & 22 & $14,01 \%$ & 8 & $10,39 \%$ \\
\hline & & \multirow[t]{2}{*}{$\boxminus 100$ a $125 \mathrm{mg} / \mathrm{dL}$} & $\mathbf{F}$ & 25 & $15,92 \%$ & 14 & $18,18 \%$ \\
\hline & & & M & 15 & $9,55 \%$ & 8 & $10,39 \%$ \\
\hline & & \multirow[t]{2}{*}{$\boxminus$ Acima $125 \mathrm{mg} / \mathrm{dL}$} & $\mathbf{F}$ & 12 & $7,64 \%$ & 14 & $18,18 \%$ \\
\hline & & & M & 8 & $5,10 \%$ & 4 & $5,19 \%$ \\
\hline & & Total & & 128 & $81,53 \%$ & 64 & $83,12 \%$ \\
\hline \multicolumn{4}{|l|}{ Total } & 157 & $100,00 \%$ & 77 & $100,00 \%$ \\
\hline
\end{tabular}

A hipertensão e o diabetes mellitusforam as patologias prevalentes encontradas como fatores não modificáveis em idosos, do sexo feminino e masculino, sendo $26,7 \%$ e $13,6 \%$; e $12,1 \%$ mulheres e $8,2 \%$ homens, respectivamente (Figuras 2 e 3 ).

Figura 2: Consulta dos fatores de risco não modificáveis agrupados por faixa etária e sexo.

\begin{tabular}{|c|c|c|c|c|}
\hline \multicolumn{5}{|c|}{ Tabela Dinâmica do Microsoft Office 11.0} \\
\hline \multirow{2}{*}{$\begin{array}{l}\text { Fator Não Modificável } \\
\text { 日Diabetes }\end{array}$} & \multirow{2}{*}{$\begin{aligned} \text { - Faixa Etária - } \\
\\
60 \text { Anos ou + }\end{aligned}$} & \multirow{2}{*}{$\begin{array}{l}\text { Sexo- } \\
\text { F }\end{array}$} & \multicolumn{2}{|c|}{ Quantidade Percentual } \\
\hline & & & 103 & $19,96 \%$ \\
\hline & & M & 70 & $13,57 \%$ \\
\hline & & Total & 173 & $33,53 \%$ \\
\hline & Total & & 173 & $33,53 \%$ \\
\hline \multirow[t]{4}{*}{ छ Hipertensão } & घ60 Anos ou + & & 227 & $43,99 \%$ \\
\hline & & M & 116 & $22,48 \%$ \\
\hline & & Total & 343 & $66,47 \%$ \\
\hline & Total & & 343 & $66.47 \%$ \\
\hline Total geral & & & 516 & $100,00 \%$ \\
\hline
\end{tabular}


Figura 3: Gráfico dos fatores de risco não modificáveis agrupados por faixa etária e sexo.

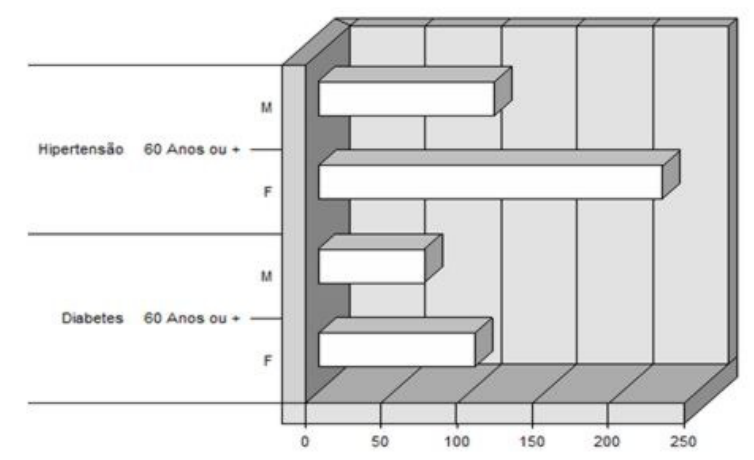

\section{DISCUSSÃO}

0 percentual representado pela população idosa nesse estudo (40,4\% indivíduos) corrobora achados da investigação com portadores de doença isquêmica em que houve predomínio do sexo feminino com maior longevidade, ${ }^{16}$ situação que pode ser justificada pelos diferentes níveis de exposição aos fatores de risco, no que se refere ao gênero, como o uso de tabaco, álcool e acidentes, levando os indivíduos do sexo masculino a mortalidade mais elevada. ${ }^{16}$ Porém, de forma controversa, há dados resultantes de pesquisa em que as mulheres apresentam maior conhecimento das doenças e seus sintomas, procurando os serviços de saúde com maior frequência, além de participarem de campanhas de promoção de saúde e prevenção de doenças; ${ }^{16}$ mas ainda assim, em nosso estudo, apresentaram predominância de hipertensão, diabetes e sobrepeso.

Diante desse fato ainda há de se ponderar o que diversos pesquisadores denominam "feminização da velhice" e suas implicações no que se refere às políticas públicas, pois uma grande parte das mulheres idosas, atualmente, é viúva, vive só, sem experiência de trabalho no mercado formal e possui baixo nível de escolaridade; ${ }^{7}$ nestes casos, a maior longevidade feminina é vista como condição preocupante, pois podem apresentar maior número de quadros senis devido às doenças crônicas quando comparadas aos homens, que falecem mais cedo.

Considerando o significativo índice de idosos hipertensos na amostra, os valores de pressão arterial elevados sugerem a necessidade de tratamento mais intensivo e controle mais rigoroso da hipertensão arterial sistêmica, uma vez que a doença está presente em um terço dos clientes da amostra, e dados da literatura referentes às aposentadorias por invalidez enfocam que a referida patologia é a mais frequente. ${ }^{16}$

Constata-se, em estudos realizados com portadores de hipertensão arterial, um dos fatores de risco para a doença arterial coronariana, que em diferentes regiões brasileiras houve aumento de sua prevalência com o avançar da idade, com predomínio no sexo feminino. ${ }^{17}$ Esta realidade encontrada em nossa investigação coincide com o relatório da Organização Mundial de Saúde ao mencionar o quadro epidemiológico atual com diminuição da mortalidade por doenças transmissíveis; ausência de políticas públicas voltadas para o atendimento a clientes com doenças crônicas, e aumento do envelhecimento populacional com a ascensão destas doenças crônicas que são responsáveis, atualmente, por $60 \%$ de todo o ônus decorrente de doenças no mundo; com previsão para 2020 de representar $80 \%$ da carga de doenças dos países em desenvolvimento.

Ressalta-se, contudo, a necessidade de ser criterioso para diagnosticar e classificar um indivíduo como hipertenso, pois o caráter multifatorial da doença, bem como a técnica de mensuração das cifras tensionais e condições de equipamentos para tal procedimento, exigem cuidados especíicos. ${ }^{13}$

0 IMC dos idosos participantes do estudo está consonante com os valores encontrados na literatura, onde se verifica que a ocorrência de sobrepeso, obesidade grau l e obesidade grau Il estão presentes entre clientes na faixa etária de 50 a 60 anos, ${ }^{16}$ idade em que comumente se dá o término de sua atividade laboral com diminuição importante das atividades exercidas em idade produtiva; assim, a intervenção no processo de aquisição de peso se faz necessária para esses clientes, através de orientações dietéticas e programas de exercícios físicos supervisionados, recomendados por especialistas, evitando que o IMC caminhe para índices mais graves. ${ }^{13} \mathrm{~A}$ obesidade foi identificada como fator de risco para a doença arterial coronariana desde o estudo de Framingham quando se constatou que o ganho de peso durante a idade adulta aumenta o risco de DAC em ambos os sexos, independente da presença de outros fatores.

Ao investigar valores de glicemia, com jejum de 8 horas, tem-se resultados preocupantes na amostra, uma vez que há elevado percentual de idosos, especialmente mulheres, com combinação de alteração de IMC, valores glicêmicos e de colesterol total enfatizando-se que o diabetes mellitus é um fator de risco significativo nas mulheres quando comparadas aos homens, pois ocasiona comprometimentos miocárdicos importantes. ${ }^{16}$

Pesquisas demonstram que o colesterol é um dos componentes para o desenvolvimento da aterosclerose que, por sua vez, leva ao aumento do risco de cardiopatias. Dados disponibilizados pela $\mathrm{OPAS}^{8}$ confirmam que 0 alto nível de colesterol no sangue causa 4,4 milhões de mortes e que a hipercolesterolemia tem maior incidência entre indivíduos com diabetes mellitus comparados aos não portadores da patologia com o mesmo grau de obesidade.

Também cabe enfatizar que a mortalidade por doença arterial coronariana pode ser decorrente, pelo menos em parte, da presença de fatores de risco modificáveis como o fumo, inatividade física, obesidade, dislipidemia e controle inadequado da hipertensão e do diabetes. ${ }^{16}$ 


\section{CONCLUSÕES}

Destaca-se a importância do controle de fatores de risco em clientes idosos portadores de doenças cardiovasculares, pois essa medida pode reduzir a exposição e minimizar, ou até eliminar, o risco de acometimento pela doença, bem como sua evolução, mesmo instalada.

Acredita-se que programas de promoção da saúde visando à identificação dos fatores de risco e tratamento adequado de indivíduos portadores de DAC podem contribuir para a redução da morbimortalidade e melhoria da qualidade de vida dos idosos, além de possibilitar o atendimento a esse novo contexto epidemiológico, em uma dinâmica de especificidades e também de assistência integral aos indivíduos, família e comunidade, considerando o gênero, especialmente o feminino. ${ }^{18}$

Desta forma, o uso de sistemas de informação que apoiam a decisão, permitindo consultas dinâmicas pelos profissionais da área da saúde, traz independência a eles para efetuarem buscas através da mineração de dados de forma fácil e flexível sem a necessidade de um especialista da área de tecnologia da informação.

\section{REFERÊNCIAS}

1.Instituto Brasileiro de Geografia e Estatística -IBGE. Projeção da população do Brasil, 2008. [on-line] [citado 2010 março 23]. Disponível em: http://www.ibge.gov.br .

2.Mendes MRSSB, Gusmão JL, Faro ACM, Leite RCBO. A situação social do idoso no Brasil: uma breve consideração. Acta Paul Enferm. 2005;18(4): 422-26.

3.Vasconcelos JF, Santos ND. Idoso e pobre na sociedade periférica capitalista: uma questão de classe social. $12^{\circ}$ Encontro de Geógrafos da América Latina e Uruguai 2009. [on-line] [citado 2010 abril 20]. Disponível em: http://egal2009.com .

4.Ministério da Saúde (BR). Secretaria da Atenção à Saúde. Departamento de Atenção Básica. Cadernos de Atenção Básica. Envelhecimento e saúde da pessoa idosa. Brasília (DF); 2006.

5.Damásio RA, Ruaro EC, Braga NQ, Nakamura EK. A enfermagem e o processo de reabilitação do idoso na sociedade. Rev Enferm Centro Universitário Andrade [on-line] 2008; [citado 20 abril 2010] 1(1). Disponível em: http://www.uniandrade.edu.br.

6.Morais ERED, Caritá EC, Silva SS."Business Intelligence" utilizando tecnologias Web para análise de fatores de risco na ocorrência de doença arterial coronariana. J Health Inform. 2010; 2 (1):8-16.

7.Camarano AA. Envelhecimento da população brasileira: uma contribuição demográfica. Rio de Janeiro(R): Instituto de Pesquisa Econômica AplicadaIPEA/Ministério do Planejamento, Orçamento e Gestão; 2002. 26p.

8.Organização Pan-Americana de Saúde-OMS. Doenças crônicodegenerativas e obesidade: estratégia mundial sobre alimentação saudável, atividade física e saúde. Brasília(DF); 2003. p. 7-56.
9.Wong LLR, Carvalho JA O rápido processo de envelhecimento populacional do Brasil: sérios desafios para as políticas públicas. Rev Bras Estud Popul. 2006; 23(1): 5-26.

10.Almeida AS, et al. Peculiaridades no tratamento da cardiopatia isquêmica no idoso. Rev Bras Cir Cardiovasc. 2007; 22(4): 476-83.

11 Moriguchi EH, Vieira JLC. Conceito de fatores de risco: hierarquia dos principais fatores de risco e susceptibilidade individual para diferentes cardiopatias. In: Giannini SD, Forti N, Diament J. Cardiopatia preventiva: prevenção primária e secundária. São Paulo(SP): Atheneu; 2000. p. 2741.

12.Braunwald E. Tratado de medicina cardiovascular. $5^{\mathrm{a}}$ ed. São Paulo(SP): Roca; 1999. v. 2. p. $1179-1460$.

13.Sociedade Brasileira de Hipertensão. Sociedade Brasileira de Cardiologia. Sociedade Brasileira de Nefrologia. V Diretrizes Brasileiras de Hipertensão Arterial. São Paulo(SP); 2006.

14.Gotto A, Assmann G, Carmena R, et al. The International Lipid Handbook for Clinical Practice. 2nd ed. New York: International Lipid Information Bureau; 2000. p.218.

15.American Diabetes Association. Standards of Medical Care in Diabetes. Diabetes Care 2005; 28 (Suppl 1): S4-S36.

16.Silva SS. Angina pectoris instável: perfil de clientes de uma instituição privada [dissertação]. Ribeirão Preto (SP): Escola de Enfermagem de Ribeirão Preto, Universidade de São Paulo; 2003.

17.Siqueira FPC. Estilo de vida e hipertensão. [dissertação]. Ribeirão Preto (SP): Escola de Enfermagem de Ribeirão Preto, Universidade de São Paulo; 2002.

18. Reza CG, Castro MEM, Solano GS, Torres DG, Martinez VG El impacto del diagnóstico: experiencia descrita por mujeres con hipertensión arterial. Esc Anna Nery 2010 jan/mar; 14 (1):33-38.

\section{NOTA}

aData Mart (DM) é subconjunto de dados de um Sistema de Apoio à Tomada de Decisão. Geralmente são dados referentes a um assunto em especial ou diferentes níveis de sumarização, que focalizam uma ou mais áreas específicas.

'OLAP é um software cuja tecnologia de construção permite aos analistas de negócios, gerentes e executivos analisar e visualizar dados corporativos de forma rápida, consistente e principalmente interativa. Sua funcionalidade é inicialmente caracterizada pela análise dinâmica e multidimensional dos dados consolidados de uma organização, permitindo que as atividades do usuário final sejam tanto analíticas quanto navegacionais 